25th AIAA Applied Aerodynamics Conference

25 - 28 Jun 2007, Miami, FL

\title{
Design, Testing and Optimization of a Constant Torque Propeller
}

\author{
Christoph Burger ${ }^{\circ}$, Roy Hartfield ${ }^{\dagger}$ \\ Aerospace Engineering, Auburn University
}

\begin{abstract}
This paper examines the viability of a passive variable pitch propeller for small aerial Remotely Piloted Vehicles (RPV's) and Unmanned Aerial Systems (UAS's) using a self adjusting mechanical pitch control mechanism. The work described in this paper includes the design and testing of a mechanical pitch change mechanism based on a constant torque spring for an off-the-shelf propeller. A propeller performance prediction code, based on the vortex lattice method, in combination with a genetic algorithm (GA) is used to optimize a propeller for given motor performance data. The optimization process includes results for both single point and multipoint design optimization efforts.
\end{abstract}

\section{Background}

Fixed pitch propellers have been used since the beginning of powered flight and are still used today because of their simplicity and weight advantage. One of the pitfalls of fixed pitch propellers is that they operate efficiently only at one propeller speed and one flight speed. Efforts on variable pitch propeller design started with the Frenchman named J. Croce-Spinelli in 1871. But it took the effort of several more inventors in different countries to bring the variable pitch propeller to aviation in the 1930's. This led to significant aircraft performance improvements with respect to cruising speed, high altitude operations and short field takeoff ability ${ }^{1}$. Later the constant speed propeller was introduced which automatically adjusts the pitch of the propeller, making it more user friendly for flight crews ${ }^{2}$. The early variable pitch propellers were mechanically operated and prone to fail after a few operating hours due to material fatigue. This led to the design of hydraulically operated variable pitch and constant speed propellers, which are still found in most of today's high performance airplanes. For more information on the development of the variable pitch propeller see Ref. 3.

\footnotetext{
* Patent applications have been filed to protect the device described in this paper.

'Graduate Student, Aerospace Eng. Dep., 211 Aerospace Eng. Bld. Auburn, AL, 36849, Member.

$\dagger$ Associate Professor, Aerospace Eng. Dep., 211 Aerospace Eng. Bld., Auburn, AL, 36849, Senior Member.
} 
The small-scale UAS's which are used in military reconnaissance operations are equipped with fixed pitch propellers which can be exchanged based on mission requirements. The reason for the use of fixed pitch propellers is system simplicity, weight savings and unavailability of a viable alternative. The design of a constant torque propeller which changes the propeller pitch automatically and without external power input based on flight condition would allow the UAS to operate at high propeller and motor efficiencies throughout the flight envelope, thus improving range, endurance or cruising speed.

Propeller performance prediction using the vortex lattice method has been proven to be a quick and accurate way of analyzing propellers ${ }^{4-7}$. Propeller design optimizations using a genetic algorithm, has been used on wind turbines ${ }^{8-10}$, ship propellers ${ }^{11}$, helicopter rotor designs ${ }^{12-13}$ and aircraft propellers ${ }^{14}$. As an integral part of developing the constant torque propeller, the blade shape is optimized in this effort using a GA driven vortex lattice code.

Both the variable pitch and constant speed propellers require a controller to operate the propeller based on aircraft flight condition. Applied to UAS's this means an additional system must be added, which adds to the overall complexity and the weight of the aircraft. The idea behind the constant torque propeller is to introduce a lightweight and passive propeller control system which requires no electric power to operate, but at the same time improves the efficiency of the motor propeller combination over the flight envelope. It can be seen from data presented in Figure 1, that if an appropriate propeller for a given electric motor (Hacker A30-10XL) is selected, high motor efficiency levels can be obtained.

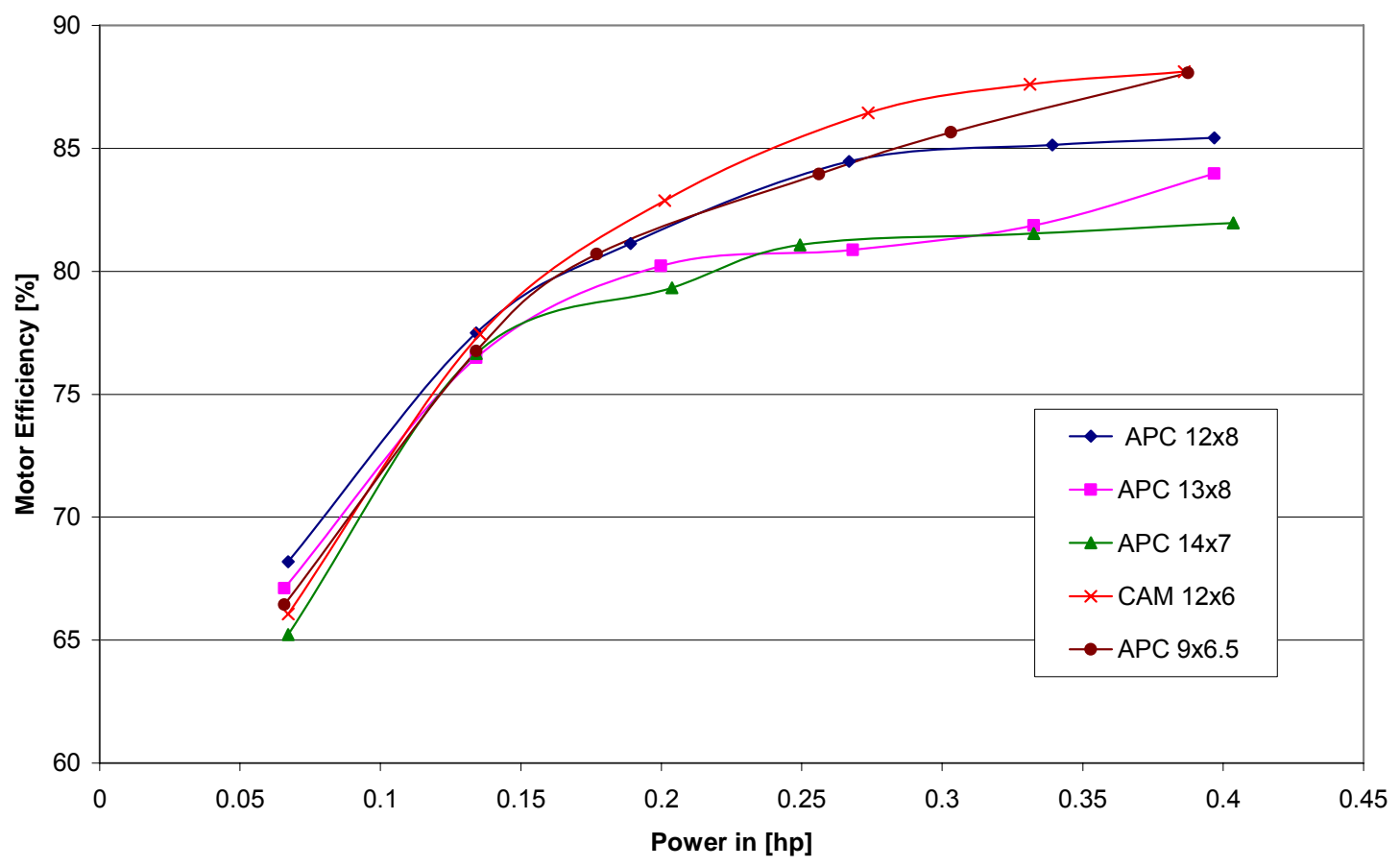

Figure 1: Power vs electric motor efficiency of a Hacker A30-10XL in static condition 
If the motor power is kept nearly constant over the entire flight envelope the operating point of the motor can be kept at a high efficiency level thus improving flight endurance and range. For the takeoff condition when higher power levels are needed motor efficiency will drop, but only for a short time until cruise condition is established.

A wind tunnel test with a variable pitch CAM $13 \times 7$ propeller was conducted to investigate maximum thrust at static conditions and at $60[\mathrm{ft} / \mathrm{sec}]$ forward flight speed. For this test, a constant power input of 67 Watts $(0.09 \mathrm{Hp})$ was maintained. Figure 2 shows a very distinct thrust peak in static condition at 1.0 [in $\mathrm{lb}_{\mathrm{f}}$ ] torque while the thrust torque slope at $60[\mathrm{ft} / \mathrm{sec}]$ velocity is rather flat with a maximum thrust at about 1.25 [in lbf]. By choosing a constant torque spring with approximately 1.0 [in lbf] the thrust can be maximized for static conditions while thrust levels in forward flight condition are maintained at close to the maximum obtainable thrust for that flight condition. Figure 2 shows also the performance of the same CAM 13x7 propeller setup in a fixed pitch mode for static conditions $(\mathrm{X})$ and for $60[\mathrm{ft} / \mathrm{sec}]$ forward flight speed $(\Delta)$.

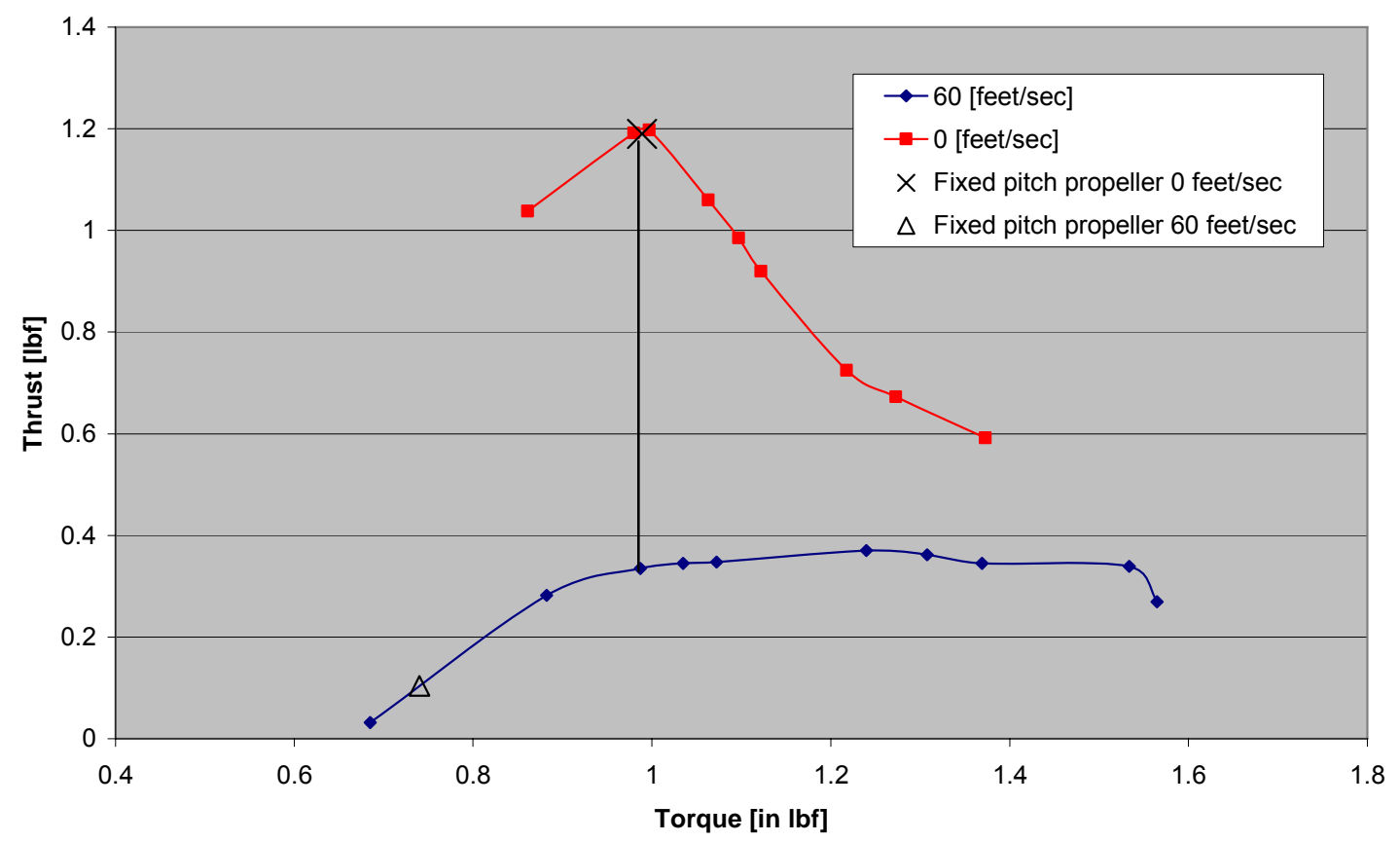

Figure 2: Propeller thrust and torque at zero and 60 [ft/sec] free stream velocity for a constant $0.09 \mathrm{Hp}$ power input

\section{Design of the Constant Torque Propeller}

For the design described in this paper, the pitch of the propeller is adjusted using a constant torque spring. The system consists of a mechanical propeller blade pitch change mechanism which uses a set of beveled gears and a constant torque spring which is 
attached to the motor shaft. See Figure 3 below. The motor attachment is connected to the propeller blade attachment via two ball bearings to allow for free rotation. The propeller blades are connected to the gear housing through an aluminum shaft which passes through a bushing with a thrust washer on the inside to allow free rotation. The inside shaft of the propeller is mounted to a beveled gear which then connects the motor shaft through another beveled gear. The torque transmitted to the propeller is dependent on the torsion between the inside shaft connected to the motor and the outside housing connected to the propeller. If a constant torque spring, to provide the torsion, is selected then a constant torque propeller assembly is the result. See Figures 4-5 for constant torque propeller assembly.

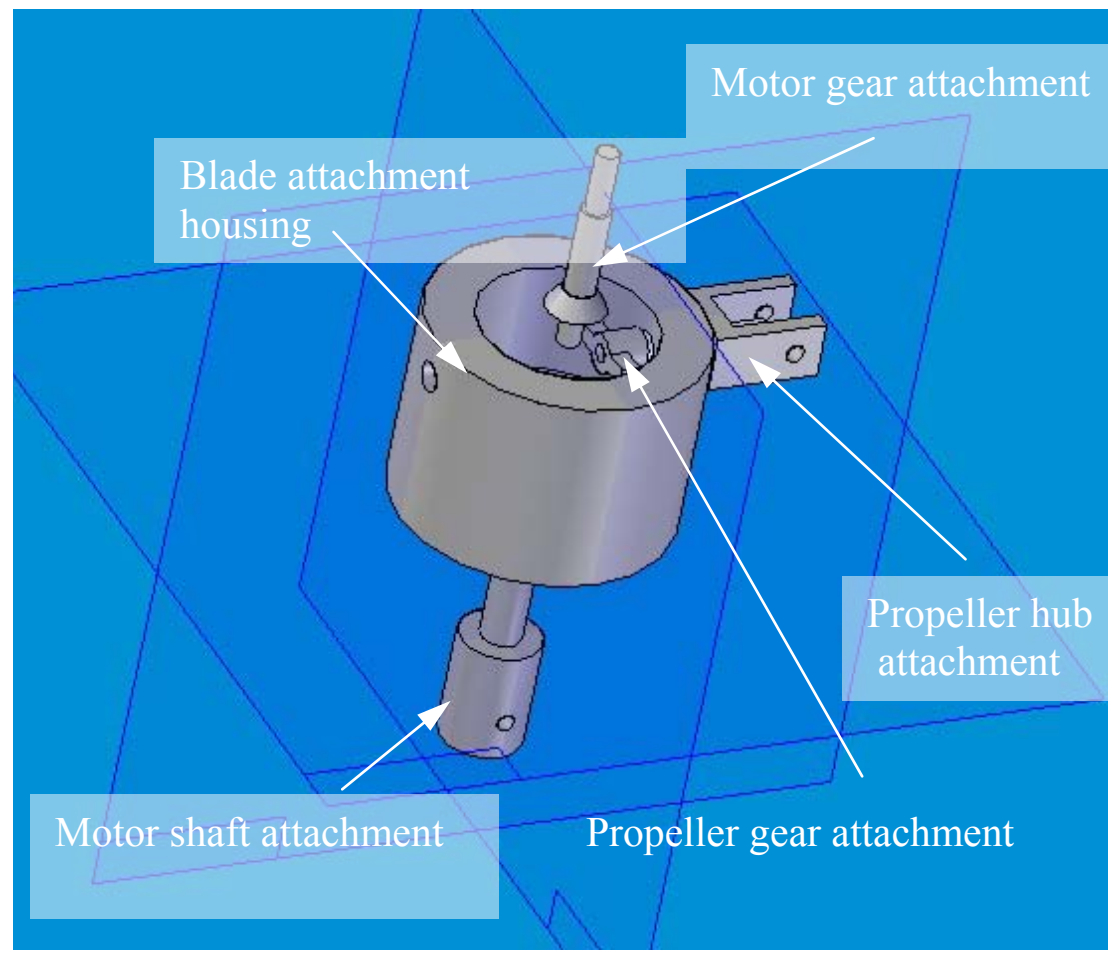

Figure 3: Constant torque propeller components

In a static condition when the propeller is not spinning the constant torque spring positions the propeller blades at the max allowable pitch setting which is limited through mechanical stops. The propeller blade assembly is arranged so that the lift of the airfoil generates a moment about the pivot line which is opposed by the moment generated by the constant torque spring. Hence, as soon as the propeller begins to rotate and the free stream velocity is kept zero, the torque builds up until it reaches the torque of the constant torque spring. This is due to the fact that when the propeller rotates, propeller lift depends on the propeller angle of attack and propeller speed. See Figure 6 below. As power continues to increase the pitch of the propeller blades is reduced to maintain the constant motor torque. In a cruise condition the load on the propeller is reduced for a fixed pitch propeller due to the increased free stream velocity, which lowers the propeller torque. For the constant torque propeller this means that as soon as the blade torque is 
reduced, the constant torque spring rotates the propeller blade to a higher pitch setting until the motor torque equals the spring torque. Any time the flight velocity changes, the constant torque spring changes the pitch of the propeller blades to accommodate torque balance.

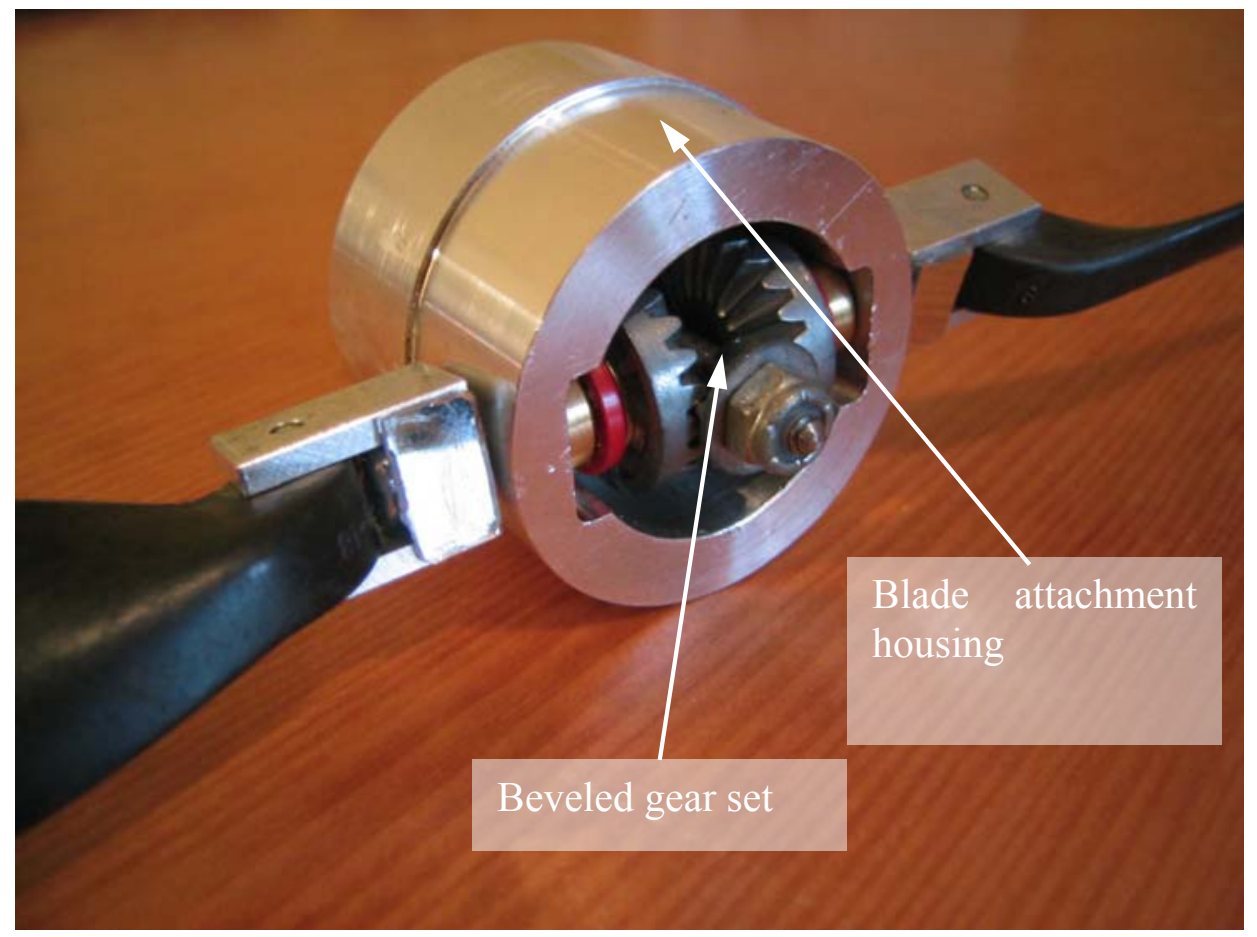

Figure 4: Constant torque propeller pitch change mechanism

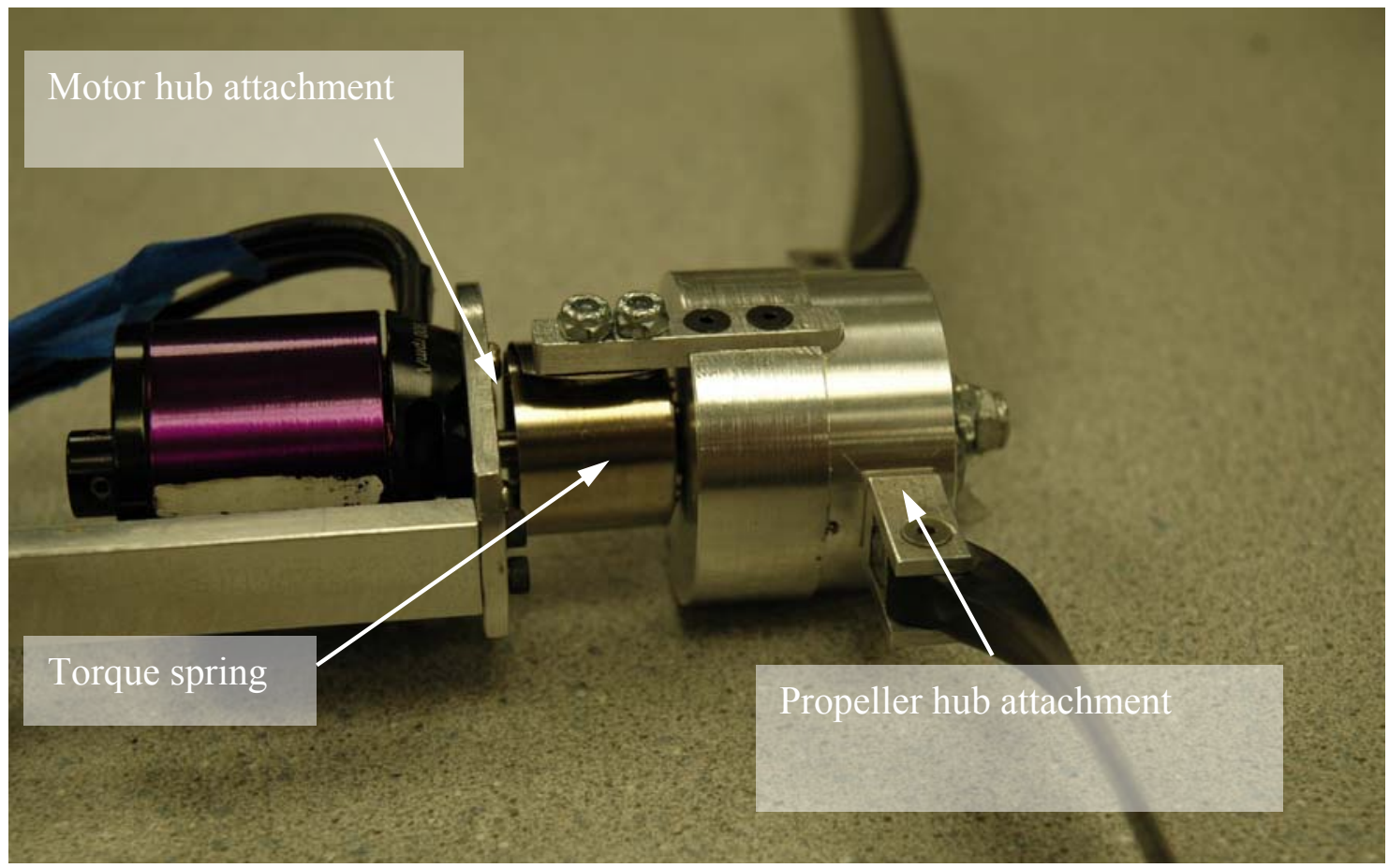

Figure 5: Constant torque propeller motor and spring attachment 


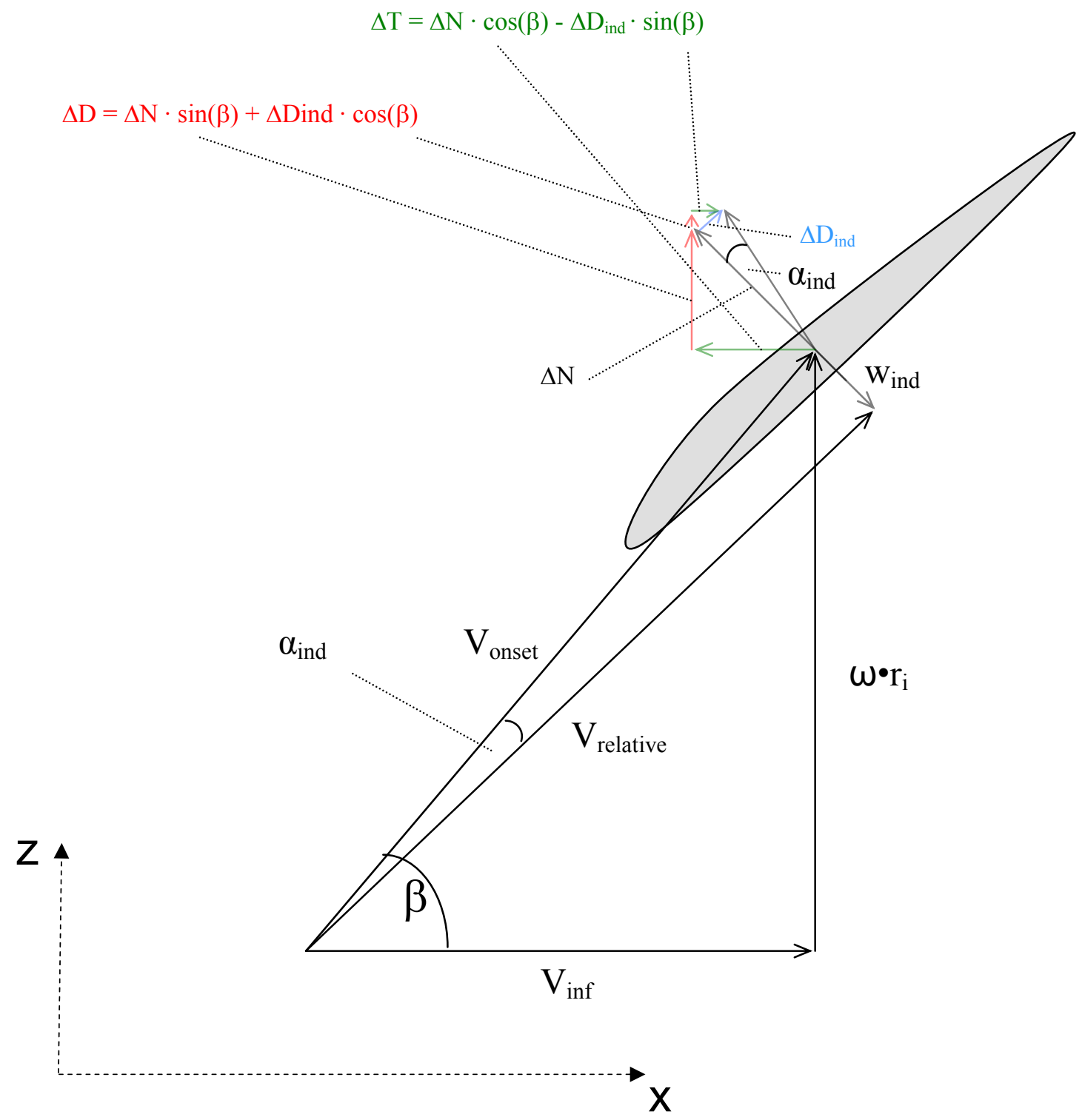

Figure 6: Propeller lift and induced drag

\section{Results}

\section{Wind Tunnel Results}

To validate the benefits of a variable pitch propeller with pitch controlled passively by the constant torque spring assembly against a fixed pitch propeller the variable pitch propeller was tested in a fixed pitch mode at a constant power setting of $\mathrm{P}=0.09$ [hp] from zero to $60[\mathrm{ft} / \mathrm{sec}]$ free stream velocity. The same setup was then tested with the variable pitch propeller at zero and at $60[\mathrm{ft} / \mathrm{sec}]$. Figure 7 shows that at zero free stream velocity the variable pitch propeller matches the thrust of the fixed pitch propeller which demonstrates that the used CAM 13x7 propeller was designed for maximum thrust at zero forward flight condition. At $60[\mathrm{ft} / \mathrm{sec}]$ free stream velocity the 
fixed pitch propeller produces about 0.1 [lbf] of thrust, while the variable pitch propeller produced 0.37 [lbf] of thrust. The max thrust data for the variable pitch propeller were obtained by changing the spring tension manually and observing the thrust produced. See also Figure 2.

Even though the variable pitch propeller produces more thrust at $60[\mathrm{ft} / \mathrm{sec}]$ free stream velocity as compared to a fixed pitch propeller, it does not reach the thrust levels of the static condition. This typical result is due to the fact that power is a function of the free stream velocity and thrust.

$$
P=T \cdot v_{\text {inf }}
$$

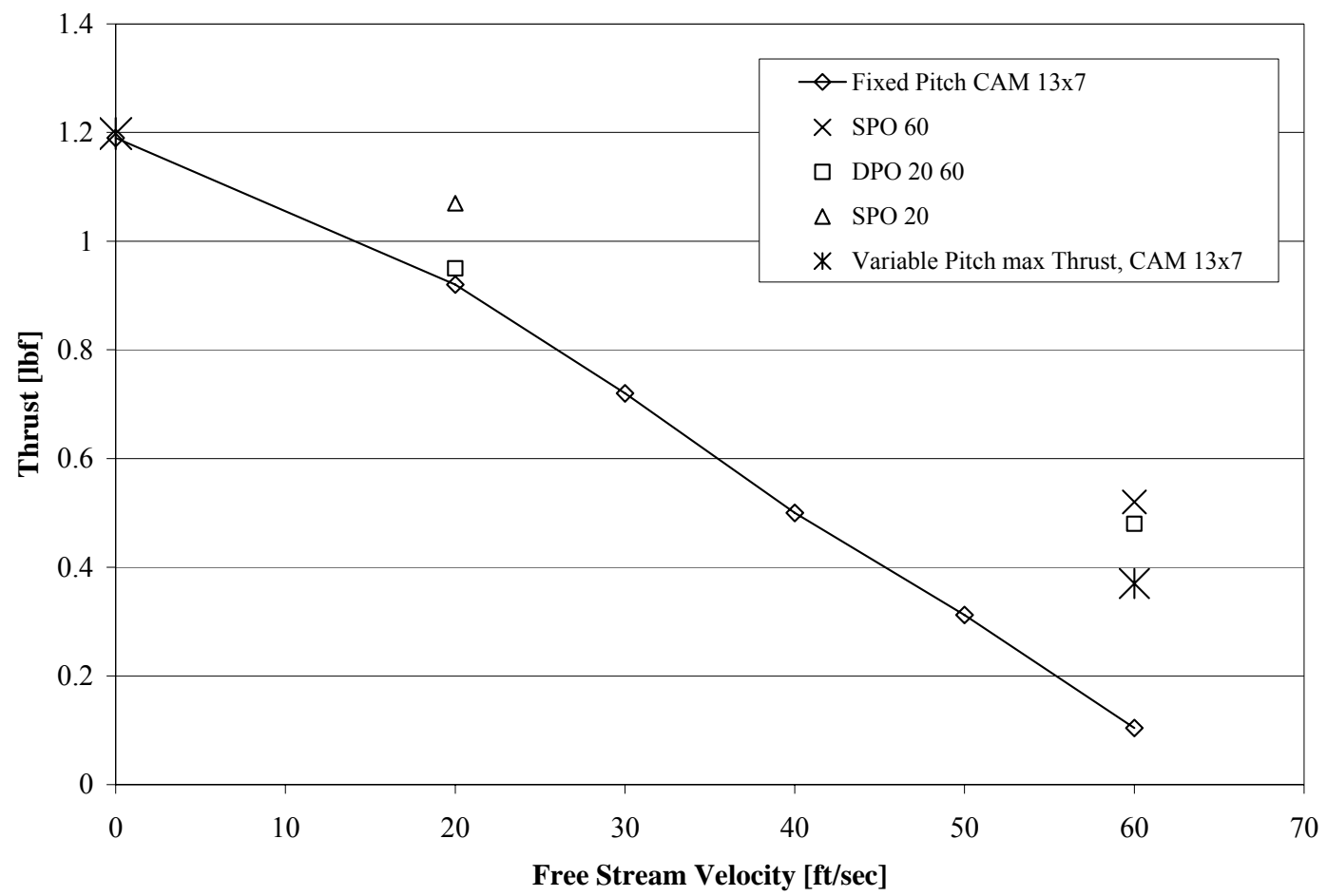

Figure 7: Thrust of a fixed pitch propeller and variable pitch propeller at $\mathbf{P}=0.9 \mathrm{Hp}$

In a second test the same CAM $13 \times 7$ propeller was tested in the wind tunnel from zero to $\sim 65[\mathrm{ft} / \mathrm{sec}]$ free stream velocity. The first test run was done in a fixed pitch propeller mode at a constant power of 0.076 [hp] and the second at the same power setting with the constant torque spring propeller setup. The performance advantage of the constant torque propeller is clearly observable in the different thrust slops of the propeller configurations of Figure 8. 


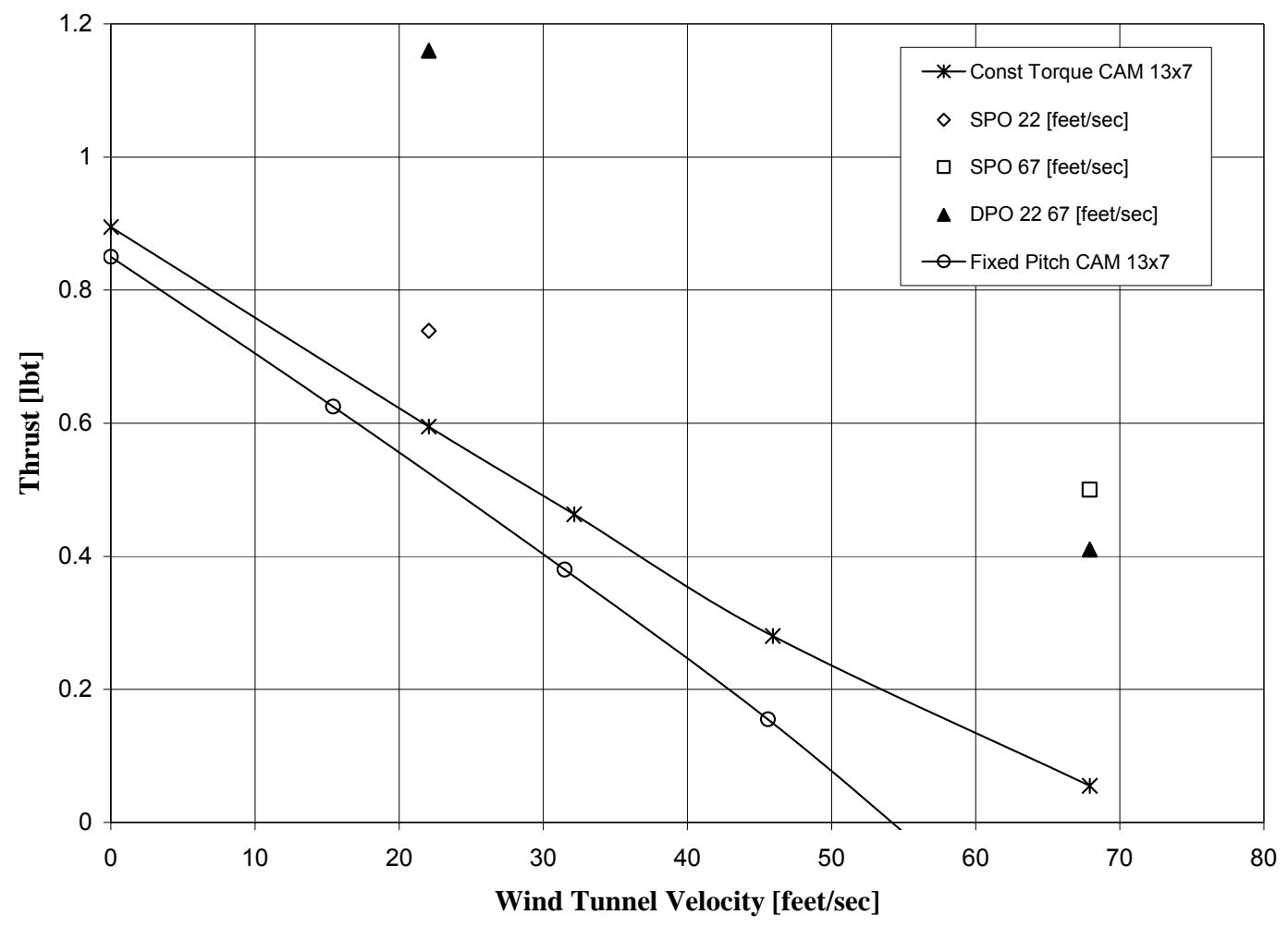

Figure 8: Fixed pitch propeller and constant torque propeller at $\mathbf{P}=\mathbf{0 . 0 7 1 - 0 . 0 7 6 ~ H p}$

\section{Propeller Performance Optimization}

To further improve the overall efficiency of the constant torque propeller, a propeller performance prediction program, based on the vortex lattice method, in combination with a genetic algorithm (GA) was used to optimize the propeller blade design. See Reference 10 and 14 for further detail on the propeller analysis program and the implementation into the GA.

For the blade shape, Bernstein polynomials and class shape functions are used to parameterize the outer mold line of the airfoil. This approach follows the method outlined in Ref. 16 and is employed such that only aerodynamic airfoil shapes are considered. No consideration is given to structural design in this study. A rudimentary flow separation model was included in this analysis; however, no viscous effects were considered.

The optimizer is a binary encoded tournament method GA with pareto capability. A population size of 400 members was used and the optimization process was carried out to several hundred generations in most cases.

\section{Optimization Results}

The CAM 13x7 propeller diameter, chord length and propeller speed was used as fixed input parameters for the optimization. The objective function was set to match a power setting of 0.09 [hp] and at the same time maximize the thrust of the propeller. Table 1 shows the thrust of the three different optimizations and the results from the 
experiments. The first and second optimizations used a single free stream velocity (SPO) of $20 \mathrm{ft} / \mathrm{sec}$ and $60 \mathrm{ft} / \mathrm{sec}$. In the third optimization, both the 20 and $60 \mathrm{ft} / \mathrm{sec}$ free stream velocity propeller operating condition was considered in the objective function (DPO). The fixed pitch CAM 13x7 propeller generates the least amount of thrust at a cruise condition of $60[\mathrm{ft} / \mathrm{sec}]$, followed by the variable pitch propeller. The best performing propellers are the SPO optimized shapes followed the DPO propeller. See also Figure 7.

Table 1: Thrust performance of different propellers at $P=0.09[\mathrm{hp}]$

\begin{tabular}{|c|c|}
\hline Propeller Type & Thrust [lbt] \\
\hline Fixed Pitch Propeller & 0.1 \\
\hline Variable Pitch Propeller & 0.36 \\
\hline 1 Optimized Propeller & 1.07 \\
\hline 2 Optimized Propeller & 0.52 \\
\hline 3 Optimized Propeller & $0.48 \quad 0.95$ \\
\hline
\end{tabular}

In a second optimization an updated version of the propeller performance program was used. The change in the program included an extended design space for the propeller geometries. See also Ref. 15. Single (SPO) and dual (DPO) point optimizations which match the power input and RPM of a second wind tunnel test were done. Even though the constant torque propeller shows superior performance as compared to the fixed pitch propeller setup, significant improvements can be obtained with an optimized propeller blade. Figure 8 shows a single point optimization (SPO) for a free stream velocity of $V_{\text {inf }}=22[\mathrm{ft} / \mathrm{sec}]$ with a thrust improvement of about $25 \%$ when compared to the constant torque propeller. At the higher free stream velocity of $67[\mathrm{ft} / \mathrm{sec}]$ the thrust improvements are even more significant. No experimental investigation has been done to maximize the thrust of the constant torque propeller setup for the power input of 0.071$0.076 \mathrm{Hp}$. The dual point optimization (DPO) for both 22 and 67 [ft/sec] free stream velocity shows that in the cruise condition the propeller thrust is lower when compared to SPO but shows significant improvements to the constant torque CAM 13x7 propeller. At the low free stream velocity the propeller performance program over predicts the propeller thrust. This is due to the fact that flow separations are not fully taken into account in the DPO run. In the SPO the flow separation is considered by limitation of the inflow (max $5 \mathrm{deg}$ ) and the downwash angle (max $10 \mathrm{deg}$ ). Figure 9 shows one of the optimized propeller geometries and wake structure. 

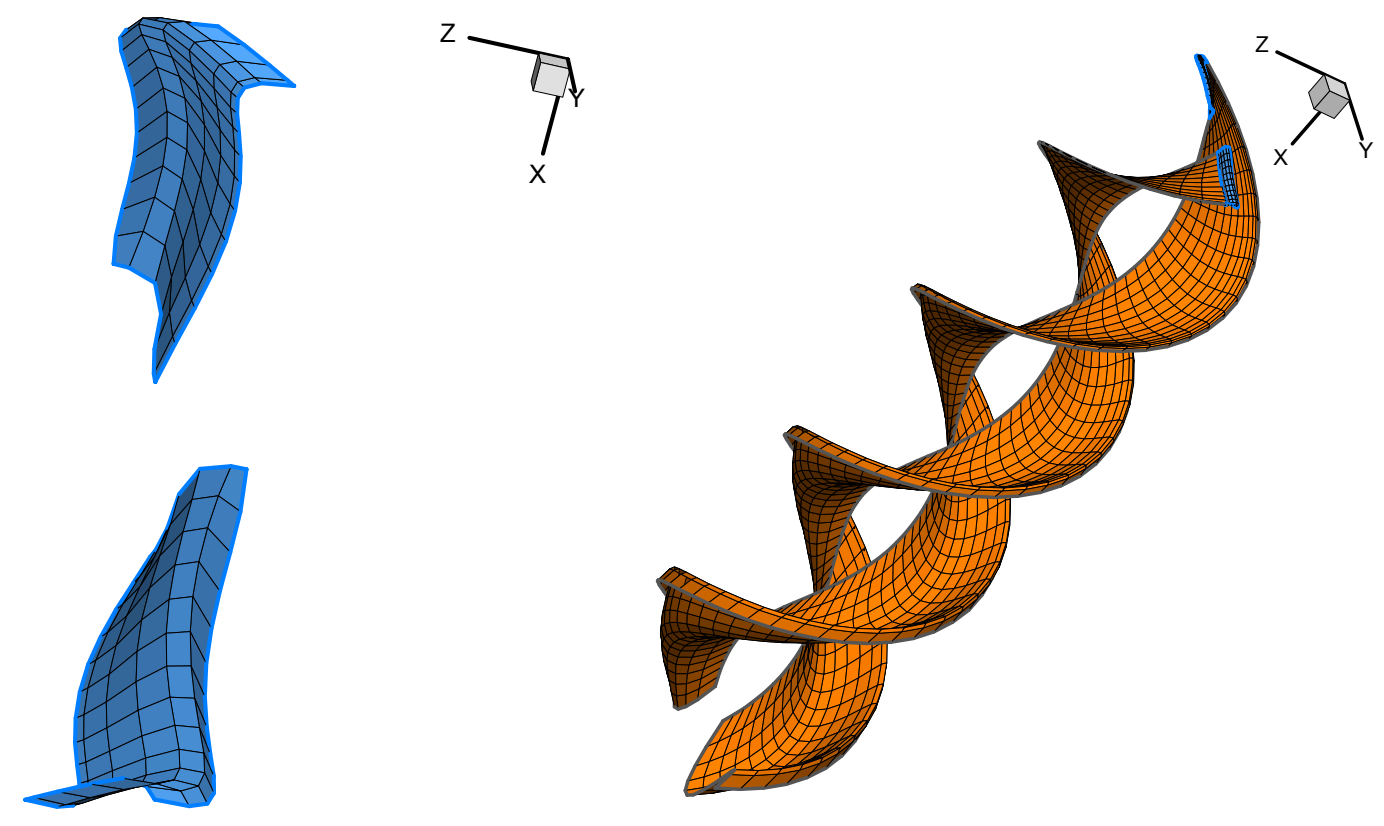

Figure 9: Optimized propeller geometry and wake structure at $V_{\text {inf }}=67[\mathrm{ft} / \mathrm{sec}]$

\section{Conclusions and Future Work}

A device which can passively practically maintain constant aerodynamic loading on propeller blades through a range of operating conditions has been designed, tested experimentally, and optimized theoretically. The experimental results demonstrate that substantial performance improvement can be obtained using this simple, passive device. The optimization results are preliminary. Planned improvements to the optimization results include the addition of a more sophisticated flow separation model, the implementation of a two-layer vortex lattice model, the inclusion of viscous effects and the addition of a cowl for certain ducted fan applications.

\section{References}

1. Fred E. Weick, "Aircraft Propeller Design", Langley Memorial Aeronautical Laboratory, McGraw-Hill Book Company, Inc. 1930..

2. Wilbur C. Nelson, "Airplane Propeller Principles", Dep. Of Aeronautical Engineering, Iowa Sate College, John Wiley \& Sons, Inc, Chapman \& Hall, Ltd 1944.

3. Jeremy R. Kinney, "Shifting gears in the air": America and the variable pitch propeller, 1928-1938, Thesis (Ph.D.), Auburn University, 2003.

4. John J. Bertin, and Michael L. Smith, Aerodynamic for Engineers, $2^{\text {nd }}$ ed.,Prentice Hall,Englewood Cliffs, New Jersey, 1989.

5. Goldstein, S., "On the Vortex Theory of Screw Propellers", Proceedings of the Royal Society of London, Series A, Vol. 123, 1929

6. Glauert, H., 1935, "Airplane Propellers," Aerodynamic Theory, ed, W. F. Durand, Julius Springer, Berlin, pp. 169-360. 
7. Shiu Wing Cheung, R. "Numerical Prediction of Propeller Performance by Vortex Lattice Method", UTIAS Technical Note No.265 Nov 1987.

8. Selig, M. S., and Coverstone-Carroll, V. L., 1996, "Application of a Genetic Algorithm to Wind Turbine Design," Journal of Energy Resources Technology, Vol. 118, March 1996, pp. 22-28.

9. Hampsey, M., 2002, "Multiobjective Evolutionary Optimisation of Small Wind Turbine Blades," PhD Dissertation, University of Newcastle.

10. Burger, C., Hartfield, R. J.,Burkalter, J. E. 'Wind Turbine Airfoil Performance Optimization using the Vortex Lattice Method and a Genetic Algorithm", AIAA 20064051, presented at the 4th AIAA Energy Conversion Engineering Conference 26-29 June 2006, San Diego, CA

11. Anders Smaerup Olsen, "Optimization of Propellers using the Vortex Lattice Method", Technical University of Denmark, Dec 2001.

12. Fanjoy D. W., Crossley W. A., "Aerodynamic Shape Design for Rotor Airfoils via Genetic Algorithm" presented at the $53^{\text {rd }}$ American Helicopter Society annual forum, Virginia Beach, April 19-May 1, 1997.

13. Jogsoo L., Prabhat H., "Parallel Genetic Algorithm Implementation in Multidisciplinary Rotor Blade Design", Journal of Aircraft Vol. 33, No. 5, SeptemberOctober 1996.

14. Burger, Christoph, Hartfield, Roy J.,Burkalter, J. E. "Propeller Performance Optimization using Vortex Lattice Theory and a Genetic Algorithm", AIAA-2006-1067, presented at the 44th Aerospace Sciences Meeting and Exhibit, Reno, NV, Jan 9-12, 2006.

15. Burger, Christoph, Hartfield, Roy J.,Burkalter, J. E. "Performance and Noise Optimization of a Propeller using the Vortex Lattice Method and a Genetic Algorithm", AIAA-2007-1883, presented at the $3^{\text {rd }}$ AIAA Multidisciplinary Design Optimization Specialist Conference 23-26 April 2007, Honolulu, Hawaii

16. Kulfan B., M., "A Universal Parametric Geometry Representation Method - CST", $45^{\text {th }}$ AIAA Aerospace Sciences Meeting and Exhibit, 8-11 January, Reno, Nevada, 2007 